\title{
Competencias Digitales Docentes y su Estado en el Contexto Virtual
}

\author{
Teaching Digital Skills and Their Status in the Virtual Context
}

\author{
ANGel Jesús Rodríguez MARTínez \\ angeljesus.rodriguez@unmsm.edu.pe \\ https://orcid.org/0000-0003-4087-7168 \\ Universidad Nacional Mayor de San Marcos
}

\begin{abstract}
RESUMEN:
En el artículo se analiza la situación actual de las competencias digitales de los docentes y su importancia para garantizar la enseñanza-aprendizaje en el contexto virtual. El método utilizado es una revisión sistemática de los documentos normativos que provienen del Ministerio de Educación del Perú y de la Unión Europea quienes abordan la problemática educativa y la educación digital, respectivamente. Entre los principales resultados tenemos que, dos de cada tres docentes presentan dificultades para sistematizar su práctica pedagógica en el contexto de la virtualidad donde solo el $1.9 \%$ de docentes crea contenidos digitales. En conclusión, casi el 98 \% de docentes se ubica en un primer nivel de dominio de las competencias digitales, haciendo uso solo a nivel elemental de las tecnologías de información y comunicación (TIC); existe la necesidad de actualizar la primera fase de la estrategia nacional de tecnología, de manera gradual y en niveles: explorador, experto, y líder.
\end{abstract}

\section{ABSTRACT:}

The article analyzes the current situation of teachers' digital skills and their importance to guarantee teaching-learning in the virtual context. The method used is a systematic review of the normative documents that come from the Ministry of Education of Peru and the European Union who address the educational problem and digital education, respectively. Among the main results we have that, two out of three teachers have difficulties to systematize their pedagogical practice in the context of virtuality where only $1.9 \%$ of teachers create digital content. In conclusion, almost $98 \%$ of teachers are located in a first level of mastery of digital competences, making use only at an elementary level of information and communication technologies (ICT); There is a need to update the first phase of the national technology strategy, gradually and in levels: explorer, expert, and leader.

\section{Palabras Clave:}

Educación digital; TIC; enseñanza virtual; competencias docentes.

\section{KEYWORDS:}

Digital education; ICT; virtual teaching; teaching skills. 


\section{Introducción}

Desde hace varios años, las competencias digitales han ido desarrollándose vertiginosamente en todo el mundo, en la misma medida que los grandes avances en el campo tecnológico, informático y comunicacional que nos ubican en un tiempo y espacio comúnmente llamado era digital; donde resulta imposible pensar en el desarrollo mundial sin tecnologías de la información y las comunicaciones (Ocaña et al., 2020). Más aún, dado el contexto sanitario producto de la pandemia generada por el COVID 19, estas competencias se perfilan como una herramienta primordial para la continuidad y desarrollo en muchas áreas del quehacer humano como: la medicina, ingeniería, finanzas, educación, entre otros (Díaz y Loyola, 2021).

En el campo de la educación, según el último informe de políticas emitido por el Grupo de las Naciones Unidas para el Desarrollo Sostenible (Gnuds) y dada la interrupción de los sistemas educativos a nivel mundial producto de la coyuntura sanitaria mundial, se vieron afectados aproximadamente 1.600 millones de estudiantes pertenecientes a más de 190 países, que al cerrarse las escuelas y diversos espacios de enseñanza perjudicando al 94 por ciento del total de la población estudiantil del mundo (Organización de las Naciones Unidas para la Educación, la Ciencia y la Cultura [Unesco], 2020). Es así que, en este contexto, las competencias digitales cobran vital importancia para garantizar el proceso de enseñanza-aprendizaje de millones de docentes y alumnos confinados en sus hogares (Gonzales et al., 2020).

En América Latina, pese a la existencia de una gran brecha digital entre los países que la conforman se menciona la necesidad de las competencias digitales en docentes para garantizar la calidad educativa. Estos deberán empoderarse de las herramientas y asumir el nuevo rol; no solo la de guía u orientador; sino la de gestor de recursos digitales (Comisión Económica para América Latina [Cepal], 2020). No obstante, los países de la región aún no han elaborado un plan común que se pueda aplicar a cada uno de los países mencionados; sin embargo, algunos países han empezado a ensayar planes pilotos de programas digitales que les permita llegar al estudiante; y así, en un breve plazo, poder cubrir la mayor cantidad de centros de estudios (Unesco, 2020).

En el Perú, tras el confinamiento, se implementó la educación remota en todo el país, evidenciando las limitaciones de los docentes del manejo tecnológico (Benavente et al., 2021). Por otro lado, el Ministerio de Educación del Perú (Minedu) ha centrado su atención en las competencias del estudiante, mientras que para los docentes no habría una política clara. Solo se están considerando documentos como el Marco de buen desempeño docente y el Diseño Curricular Básico Nacional (DCBN) que orientan de manera general el uso de los recurso tecnológicos; de ahí que emprender la restructuración digital de la formación docentes y estudiantes del Perú, demandaría varios años (Trahtemberg, 2021). En ese sentido, el objetivo general de la presente investigación es analizar el estado de desarrollo de las competencias digitales de los docentes en el Perú en el contexto de la virtualidad para poder proyectarse en un nuevo escenario de enseñanza virtual derivado de la pandemia.

\section{Competencias digitales de los docentes}

La competencia digital se puede entender como la capacidad para utilizar los diferentes recursos digitales y herramientas de manera adecuada; para ello, se pasa por un complejo y gradual proceso de aprendizaje; desde la búsqueda de información hasta la transformación de la misma de forma crítica (Perdomo et al., 2020). Ello implica poder utilizar la tecnología digital de manera consciente y críticamente (Sá y Serpa, 2020). De igual forma, Solís y Jara (2019), sostienen que el desarrollo de dicha capacidad debe estar en el proceso de aprendizaje diario, dado el inherente avance de la sociedad digital, que en muchos aspectos es determinante para el éxito de cada individuo.

Krumsvik et al. (2018) plantean una visión holística de las competencias digitales, donde se encuentran capacidades y saberes del ámbito tecnológico nacidas desde un nivel superior, con un sustento en una alfabetización digital de naturaleza funcional. De ahí que se necesitan nuevas competencias para un nuevo 
contexto, nuevas habilidades y actitudes para el desarrollo de la alfabetización digital, acompañado de un plan novedoso que garantice dicho objetivo (Levano et al., 2019).

En cuanto al docente se refiere, esta competencia digital se concibe como una relación tripartita de actitudes, conocimientos y habilidades necesarias para el empoderamiento de las TIC como herramientas para su práctica pedagógica diaria (Cabero y Martínez, 2019). Ello implica un cambio de rol del docente, dada la abundante información en la red, donde deberá sistematizar el conocimiento: accediendo, seleccionando, evaluando para finalmente transmitir el conocimiento en su actividad pedagógica, complementando la orientación y guía del estudiante (Díaz y Loyola, 2021).

\section{Dimensiones de las competencias digitales docentes}

En el Marco Europeo de Competencia Digital (DigCompEdu), se consideran seis competencias digitales para los docentes. En el número uno se contempla el compromiso personal; el número dos, los recursos digitales; en el número tres, la pedagogía digital; en el número cuatro, la evaluación y retroalimentación; en el número cinco, el empoderamiento a los estudiantes, y en el número seis, la facilidad para la competencia digital de los estudiantes. La primera área competencial, el compromiso profesional docente, constituye una herramienta de comunicación, fundamental para el docente debido a que le permite interactuar digitalmente con colegas, alumnos y padres de familia; usando los diferentes canales digitales como sitios web, WhatApp, blogs, entre otros. Asimismo, esta primera área le permite al docente una práctica reflexiva y una formación digital autónoma a través de cursos online de administración, mooc, webinars, entre otros (Cabero et al., 2020).

Siguiendo a Caena y Redecker (2019), el dominio de estas seis competencias digitales o áreas de desarrollo se toman en cuenta para clasificar y ubicar al docente, según el manejo que tenga de estas, en tres niveles: Así tenemos, un primer Nivel A, donde se encuentran los docentes que recién se inician en la exploración de las tecnología buscando su aplicación en el proceso de enseñanza; ya en un segundo Nivel B, tenemos a los docentes integrador - expertos, que usan las herramientas de diferentes maneras y en múltiples contextos pedagógicos; finalmente, un tercer Nivel C, donde se ubican los líderes e innovadores, los docentes empoderados de las competencias digitales; ellos conocen las diferentes herramientas, enfoques y crean nuevos métodos y estrategias pedagógicas de aprendizaje y evaluación,siendo esta última, donde el docente experimenta con nuevas formas tecnológicas y se proyecta a un contexto diferente (Díaz \& Loyola, 2021).

\section{Dimensiones de las competencias digitales para el docente latinoamericano}

Según la propuesta de Rangel (2014), el perfil del docente de la región debe estar compuesto básicamente por tres dimensiones: tecnológico, informacional y pedagógica; y a su vez formada por trece competencias con sus respectivos indicadores cada una. Se plantea así, una propuesta que enfatiza el papel del docente, dejando relegado la del estudiante, personaje importante que se debería buscar beneficiar para elevar su aprendizaje (Díaz y Loyola, 2021).

\section{Importancia de las competencias digitales}

Las competencias digitales docentes cobran gran importancia dado el contexto pandémico, en la participación de las personas de los diferentes sectores de la sociedad; económico, social, educativo, entre otros (Sá y Serpa, 2020). En este último sector, las TIC aparecen como una forma de desarrollo, con una gran posibilidad de éxito en alcanzar una mayor interactividad con el estudiante; así también aparece como un mecanismo de generación, transmisión y evaluación en la actividad educativa y la construcción del aprendizaje significativo (Zárate, et al., 2020).

Las competencias digitales cumplen un papel fundamental en el actual contexto (Linares et al., 2018), teniendo en cuenta que el mayor porcentaje son estudiantes identificados como la generación del milenio. A ellos se les atribuye diversas características digitales y opiniones, que muchas veces son opuestas al docente en cuanto a la forma y contenido del proceso de enseñanza-aprendizaje. De ahí, la importancia de contar con un 
docente capacitado en TIC, que acorte la brecha generacional, en cuanto a las competencias digitales se refiere y poder cubrir las necesidades de los estudiantes (Cobos, et al., 2018). Debido a que un docente con un escaso manejo tecnológico solo se limita a la asignación de actividades domiciliarias evidenciando el uso tradicional de las competencias digitales, de ahí el reto de revertir tal escenario (Benavente et al., 2021).

Según la investigación realizada por Martínez y Garcés (2020) sobre las competencias digitales del docente frente un programa enseńanza e-learning, correspondiente a una institución educativa de Colombia; se concluye que la capacidad más desarrollada, por parte de los docentes, es la informatización y alfabetización digital, mientras que creación de contenidos digitales es una de las competencias más débiles de los mismos. Recomendando la implementación de un programa de fortalecimiento de las competencias en todas sus dimensiones como estrategias para alcanzar una enseñanza de calidad dentro del contexto sanitario producto de la COVID-19.

Por otra parte, el estudio realizado por Díaz y Loyola (2021), sobre la revisión documental acerca de las últimas conceptualizaciones de las competencias digitales del docente bajo el contexto de la pandemia, concluye que una de las estrategias para el desarrollo de la enseñanza virtual es el modelo del Aula Invertida, sustentado en la información de los estudios revisados.

Cabe añadir también que, uno de los principios de la Estrategia Nacional de las tecnologías digitales en la educación básica 2016-2021, es el rol del docente como agente de cambio. Se concibe ello, debido que con el empoderamiento de las tecnologías digitales, el docente podrá cumplir con su rol de generador de cambio; impulsando las TIC desde el aula física o virtual, prepararse para los cambios y desafíos del aprendizaje y del trabajo colaborativo con el estudiante; pese a estar, este último, mayor familiarizado con lo digital (Ministerio de Educación, 2016).

Asimismo, Holguin et al. (2021) realizaron un estudio sobre las competencias digitales en los directivos y docentes en un contexto de aprendizaje remoto, producto de la emergencia sanitaria en el Perú; donde se concluyó que los docentes de las instituciones educativas seleccionadas para la muestra tienen un mayor nivel de competencias digitales con respecto a los directivos; resaltando el dominio para la comunicación, colaboración y creación de recursos digitales de los primeros. Finalmente, en el informe de políticas educativas durante la COVID-19 publicado por la ONU (Organización de las Naciones Unidas, 2020), recomienda apoyar la preparación de los docentes para afrontar dicho contexto, a fin de asegurar el aprendizaje equitativo e inclusivo en cualquier medio.

\section{Método}

El propósito del presente estudio fue realizar una revisión documental acerca del estado y el desarrollo de las competencias digitales de los docentes en un contexto de confinamiento. La investigación se desarrolló bajo el enfoque cualitativo, que según Arriaga (2013), se fundamenta en la comunicación de las personas y los grupos sociales lo que sirven de base para establecer un diálogo intersubjetivo social, y primordial tanto para la generación como para el desarrollo de un nuevo conocimiento. El tipo de investigación es básica porque se busca sistemáticamente un conocimiento nuevo que amplíe la concepción de la realidad concreta (Álvarez et al., 2017). El diseño se realizó mediante una revisión sistemática de la literatura, que según Linares et al. (2018), implica una síntesis crítica y publicables de los resultados de las investigaciones disponibles sobre una misma temática.

Se siguió la técnica del análisis documental, empleando las bases de datos Scopus, Web of Science, Scielo; usando el gestor bibliográfico Mendeley para sistematizar los resultados obtenidos. La selección de los artículos de investigación se hizo tomando en cuenta los criterios: las publicaciones que contengan al menos una de las palabras claves y a su vez que corresponda a los cinco ańos de antigüedad. La muestra seleccionada corresponde a cuatro documentos publicados por el MINEDU y uno por la Comunidad Europea que trazan 
la política educativa digital de la educación básica regular. La recolección de los datos se realizó a través de fichas de análisis documentales.

\section{Resultados}

Resultado del análisis del documento: Estrategia nacional de las tecnologías digitales en la educación básica 20162021 (Ministerio de Educación, 2016)

\section{- Acerca del hito estratégico para la mejora de la competencia digital docente}

En dicho documento, se planea que el empoderamiento de las competencias digitales (CD) permite aumentar el desempeño docente, dentro del Marco de Buen Desempeño Docente (MBDD), y con ello mejorar las habilidades y estándares del aprendizaje planificado haciendo uso del factor tecnológico. Asimismo dicho plan contempla seis fases o hitos que forman parte de la estrategia nacional digital docente de educación básica 2016-2021, donde se considera el nuevo rol del docente como agente de cambio. Con este empoderamiento de las tecnologías digitales, el docente podrá cumplir nuevas funciones; impulsando las TIC desde el aula física o virtual, prepararse para los cambios, desafíos del aprendizaje y del trabajo colaborativo con el estudiante, pese a que este último está más familiarizado con lo digital. Para ello, el docente tendrá como herramienta de gestión una matriz de competencias digitales donde se identifiquen y registren las habilidades digitales, así como el uso de las áreas de información, generación de conocimientos, innovación, entre otros.

Las seis fases o hitos contemplados en el plan estratégico tuvieron los siguientes objetivos: en la fase preparatoria se proyectó desarrollar las estrategias y herramientas adecuadas; luego en el año 2017 se buscó ejecutar el Hito 1, cuyo objetivo fue garantizar la capacitación digital de los docente; en el año 2018, ejecutar el Hito 2, cuyo fin fue dotar a las escuelas de un kit digital; en el año 2019, el Hito 3, donde se buscó el uso a nivel nacional de la conectividad en los diferentes contextos del Perú; en el año 2020, el Hito 4, centrar el desarrollo de las capacidades digitales del estudiante, para finalmente, en el años 2021, desarrollar en el Hito 5 , donde todo los actores del proceso de enseñanza-aprendizaje adquieren la cultura digital. (MINEDU, 2016).

\section{- Acerca del Hito 1 para el desarrollo de la competencia digital docente}

Hasta el 2017, se proyectó la primera fase o hito que consistía en la familiarización del docente con las herramientas digitales necesarias para su práctica pedagógica, para lo cual se planificaron diferentes acciones como:

- Acciones de logro que poseían dos etapas, la primera corresponde a la sensibilización, donde se buscó familiarizar al total de los docentes con el uso tecnológico digital para luego empiece a emplear en el proceso de enseńanza; sin embargo, los resultados encontrados fueron que el $70 \%$ de docentes cuenta con alguna capacitación; mientras que, un $8 \%$ se ubica en el nivel integrador de tecnología en el aula; además, existe un $22 \%$ de docentes que no tienen ninguna capacitación de las TIC. Cabe resaltar que dichos logros solo han apuntado al uso del dispositivo y no al desarrollo de las competencias docentes. La segunda etapa desarrollada es el Programa para TIC, que busca capacitar al $100 \%$ de docentes, pero centrándose en el desarrollo de las competencias, empezando con el porcentaje educadores que no poseen ninguna capacitación.

- Acciones de Diseño que definió el desarrollo de las competencias digitales docentes a partir de una matriz de competencia digital donde se considera tanto al uso del dispositivo tecnológico como el desarrollo de las habilidades digitales; sobre la base de dicha matriz, juntamente con los objetivos anuales propuestos, se construirá una herramienta fundamental para los diferentes momentos pedagógicos del educando. La otra etapa de la acción de diseño fue la creación de la plataforma 
virtual PeruEduca, elaborada con el objetivo de ser un espacio de interacción entre todos los actores del proceso educativo, donde se formulen propuestas, se desarrolle el trabajo colaborativo proyectándose más allá de la institución educativa.

- Acciones de experimentación que conllevaron al desarrollo de la plataforma digital para la formación de los docentes y a la contrastación de su práctica para el desarrollo de los niveles de familiarización hasta la especialización, teniendo en cuenta el CN y el MBDD, la contrastación docente y la contrastación en el campo de los procesos formativos.

\section{Resultado del análisis del documento: Diseño Curricular Básico Nacional del Perú (DCBN)}

- Acerca de los criterios básicos para el desarrollo de la competencia digital docente en su formación inicial.

Siguiendo el DCBN (Minedu, 2015), la formación docente implica un desarrollo de la competencia digital del docente de manera transversal en todos los cursos de su formación inicial. Para ello, plantea cuatro criterios que orientan la integración y desarrollo de las competencias digitales: la gestión de la información en entornos digitales; a través de esta capacidad, el docente dinamiza la sistematización de la información usando los entornos digitales con eficacia, analizando el contenido académico de manera crítica, con responsabilidad y ética. Dentro de este proceso de sistematización se encuentran el saber discriminar, organizar y distribuir la información académica respetando la propiedad del autor.

Segundo, la gestión de la práctica pedagógica utilizando herramientas y recursos en los entornos digitales; el docente deberá usar todas las herramientas digitales educativas de su entorno de manera autónoma y crítica en el desarrollo de proceso de enseñanza-aprendizaje a través del trabajo colaborativo, creando nuevos formatos de contenidos de acuerdo con las características propias de cada estudiante. La tercera es la utilización de entornos y redes digitales; con esto el docente socializará los conocimientos a través de una comunicación mucho más amplia y democrática ya sea a través de foros, chats, entre otros. Y el número cuatro que es referido al empleo de recursos en los entornos digitales como herramientas de los aprendizajes, esto permitirá evaluar al estudiante virtualmente de manera sincrónica y asincrónica. Así, estos cuatro aspectos permitirán la formación de un docente con mayor eficiencia, sentido crítico, a partir del uso de las herramientas y recursos educativos.

\section{Resultados del análisis del documento: Encuesta Nacional a Docentes de instituciones educati- vas públicas de la educación básica regular- ENDO REMOTA 2020, Minedu 2021}

- Acerca de la percepción del docente sobre tecnología para la educación en el contexto sanitario de la pandemia.

Según la encuesta ENDO REMOTA correspondiente al año 2020; el 69.6\% de los docentes tuvieron dificultad para sistematiza su práctica pedagógica. Asimismo, el 65.2\% de los docentes a nivel nacional, valora su desempeńo durante la pandemia, catalogándolo como un rol importante para el desarrollo del Perú. Además, el 67.0\% de docentes se capacitó en el uso de las herramientas tecnológicas; el 83.0\% participó en diferentes cursos virtuales; el $92.1 \%$ considera de muy útil la capacitación recibida para su trabajo; y el $83.4 \%$ de los encuestados considera que el acceso a la tecnología e internet ayudaría a mejorar su práctica docente.

Resultados del análisis del documento: Educación digital plan acción 2021-2027 - Comunidad Europea

- Acerca de formación digital docente y el nuevo contexto

En dicho documento se resalta la competencia digital, como elemento necesario e indispensable para todos los docentes debido a que dicha habilidad le sirve para innovar, involucrar y motivar al alumno, 
apoyando a este último en su desarrollo digital. De ahí que la competencia digital debe ser incluida en todas las áreas y programas de desarrollo de los educadores. Asimismo, los programas deben ser libres y continuos, teniendo en cuenta las características de cada disciplina o curso. El documento también propone una categorización de los profesores, de acuerdo con el desarrollo de las habilidades digitales de cada uno de ellos. Así en un nivel A (novato, explorador), se encontrarán docentes que recién empiezan a usar los dispositivos tecnológicos; seguidamente, en un nivel B (integrador y experto), están los docentes que logran integrar lo tecnológico en diferentes contextos de su vida profesional y familiar; finalmente se encontrarán en el nivel C (líder e innovador), los docentes que usan tecnologías y plataformas más complejas buscando innovar la enseñanza del alumno. Finalmente, se busca garantizar el aprendizaje virtual con docentes expertos, líderes e innovadores.

\section{Resultado del análisis del documento: Marco de Buen Desempeño Docente}

- Acerca del uso de recursos y tecnologías por parte del docente

En el análisis del documento se encontró que, según el dominio dos, competencia cuatro y desempeño 23, del documento en mención, es el docente quien guía el desarrollo del proceso de la enseñanza haciendo uso de los diversos recursos y herramientas tecnologías accesibles, tanto para el docente como para el alumno, para el desarrollo de cada sesión de aprendizaje. Asumiendo así un papel de gestor y mediador tecnológico: creando, seleccionando y organizando los recursos digitales, base y soporte para el aprendizaje de los estudiantes; teniendo en cuenta los estilos y nivel de cada uno de ellos. Asimismo, el docente enfatizará en un aprendizaje reflexivo y crítico del alumno, para que las herramientas tecnológicas le sirvan para la solución no solo de su vida académica sino también en el contexto diario.

\section{Discusión}

En la presente investigación se planteó como objetivo realizar un análisis documental de las competencias digitales de los docentes y poder describir el desarrollo de estas en un posible escenario de enseńanza post pandemia. En ese sentido, en el documento primario, Marco de Buen Desempeño Docente, plantea el uso de las competencias digitales como una necesidad para guiar la enseñanza del alumno; describiendo el papel de un gestor tecnológico asignado al docente (Minedu, 2014).

Dicha necesidad de empoderamiento digital por parte del profesor, también se percibe en la Estrategia Nacional de las tecnologías digitales en la educación Básica 2016-2021; donde el Ministerio de Educación proyectó que para al término del año 2017, se cubriría con la capacitación en herramientas digitales del 100\% de profesores a nivel nacional (Minedu, 2015). Considerando para este objetivo varias etapas como: sensibilización, diseño, implementación y experimentación; sin embargo, según los resultados de las encuestas ENDO 2018, solo el 27.1\% de docentes recibieron capacitación en el uso de las tecnologías de la información durante el año 2017; evidenciando que no se cumplió con la meta del $100 \%$, proyectada en la estrategia nacional.

La presente investigación coincide con la necesidad de impulsar y desarrollar las competencias digitales en la práctica pedagógica docente, como gestor y creador de contenidos digitales planteadas en el Diseño del Currículo Básico Nacional (Minedu 2016). Esto se evidencia en la encuesta ENDO REMOTA 2020, donde el $69.9 \%$ de profesores tuvieron dificultad para sistematizar su práctica pedagógica. De ahí, la necesidad de impulsar el empoderamiento de estas habilidades digitales, por parte de los docentes; de lo contrario, solo estarían haciendo el uso tradicional de las TIC, como la asignación de tareas domiciliarias (Benavente et. al. 2021). Esto podría tener concordancia con los resultados obtenidos por Martínez y Garcés (2020) donde se encontró que solo el 1,9\% de docentes crean contenidos digitales, mientras que el porcentaje restante se acercan a la primera etapa que es la digitalización y alfabetización digital. 
Asimismo, se encontró que el desarrollo de las competencias digitales por parte de los docentes implica un proceso complejo y gradual (Perdomo y Gonzales, 2020); la misma óptica posee la Estrategia Nacional de Tecnología (Minedu 2016). De ahí que la política ministerial planificó el desarrollo de las competencias de los docentes dentro del programa macro comprendido en seis fases o hitos estratégicos para un lapso de seis años (2016-2021). Donde el hito 1, correspondió al fortalecimiento de las competencias digitales docentes desarrollado durante el año 2017; dentro de las acciones de logro ejecutados en ese periodo fue la elaboración de la plataforma digital PeruEduca.

Las capacitaciones y desarrollo de las habilidades digitales en los docentes se aceleraron por motivo de la pandemia y la enseñanza virtual, reflejándolo en el ENDO 2020 (Minedu, 2020), que dio como resultados que un $83.0 \%$ de profesores del Perú, participaron en cursos virtuales y un $67.0 \%$ se capacitaron en el uso de las TIC. Este escenario conllevo a que la mayoría de los docentes tuvieran un acercamiento a las herramientas digitales; sin embargo, si se compara con algunos estándares de la región, estaría faltando elevar el grado y nivel del dominio docente a un grado de experto y líder con la posibilidad de garantizar el éxito de una enseñanza virtual en este contexto pandémico.

\section{Conclusiones}

Se puede concluir que la mayor cantidad de docentes, 98\% aproximadamente, se encuentra en una primera etapa del desarrollo de las competencias digitales, haciendo uso solo a nivel de las TIC. Asimismo, la necesidad de fortalecer las acciones de logro del plan estratégico nacional de las tecnologías, a través de un programa de capacitación digital docente centrado, no solo en uso del dispositivo, sino también en el desarrollo de todas las dimensiones de las competencias digitales, es decir, no solo en el uso del hardware sino también en la aplicación y uso del software. Esto con la finalidad de permitirle al docente gestionar su práctica pedagógica con herramientas, recursos y entornos digitales durante la virtualidad con proyección a un contexto post pandemia. Finalmente, actualizar la fase uno de la estrategia nacional de tecnología, buscando capacitar a la mayor cantidad de docentes de manera gradual, manejando tres niveles: explorador, experto y líder, necesarios para garantizar el rol del docente como gestor y mediador digital.

\section{Referencias}

Álvarez, E. P., Núñez, P., y Rodríguez, C. (2017). Adquisición y carencia académica de competencias tecnológicas ante una economía digital. Revista Latina de Comunicacion Social, 72, 540-559. https://doi.org/10.4185/RLCS-20171178

Benavente, S. Ú., Flores, M., Guizado, F., y Núñez, L. A. (2021). Desarrollo de las competencias digitales de docentes a través de programas de intervención 2020. Propósitos y Representaciones, 9(1). https://revistas.usil.edu.pe/index. php/pyr/article/view/1034/1308

Caena, F. y Redecker, C. (2019). Aligning teacher competence frameworks to 21st century challenges: The case for the European Digital Competence Framework for Educators. https://onlinelibrary.wiley.com/doi/full/10.1111/ejed.12345

Cabero, J., Barroso, J., Palacios, A., y Llorente, C. (2020). Marcos de Competencias Digitales para docentes universitarios: su evaluación a través del coeficiente competencia experta. Revista Electronica Interuniversitaria de Formacion Del Profesorado, 23(2), 1-18. https://doi.org/10.6018/reifop.413601

Cabero, J., y Martínez, A. (2019). Las TIC y la formación inicial de los docentes. Modelos y competencias digitales. Profesorado, Revista de Currículum y Formación del Profesorado, 23(3), 247-268. https://doi.org/10.30827/ profesorado.v23i3.9421

Díaz, D., y Loyola, E. (2021). Competencias digitales en el contexto COVID 19: una mirada desde la educación. Revista Innova Educación, 3(1), 120-150. https://doi.org/10.35622/j.rie.2021.01.006

Krumsvik, R. J., Berrum, E., \& Jones, L. Ø. (2018). Everyday digital schooling - Implementing tablets in Norwegian primary school: Examining outcome measures in the first cohort [Educación digital cotidiana: implementación 
de tabletas en la escuela primaria noruega: examen de las medidas de resultado en la primera cohorte], Nordic Journal of Digital Literacy, 13(3). https://doi.org/10.18261/ISSN.1891-943X-2018-03-03

Levano, L., Sanchez, S., Guillén, P., Tello, S., Herrera, N., y Collantes, Z. (2019). Competencias digitales y educación. Propósitos y Representaciones, 7(2), 569-588. https://doi.org/10.20511/pyr2019.v7n2.329

Linares, E., Hernández, V., Domínguez, J. L., Fernández, S., Hevia, V., Mayor, J., Padilla, B., \& Ribal, M. J. (2018). Methodology of a systematic review [Metodología de una revisión sistemática], Actas Urológicas Españolas (English Edition), 42(8), 499-506. https://doi.org/10.1016/j.acuroe.2018.07.002

Martínez, J., y Garcés, J. (2020). Competencias digitales docentes y el reto de la educación virtual derivado de la covid-19. Educación y Humanismo, 22(39), 1-16. https://doi.org/10.17081/eduhum.22.39.4114

Ministerio de Educación del Perú. (2014). Marco de Buen Desempeño Docente. Para mejorar tu práctica como maestro y guiar el aprendizaje de tus estudiantes. https://bit.ly/3iQjuWv

Ministerio de Educación del Perú (2015). Diseño Curricular Básico Nacional de la Formación Inicial Docente - Programa de estudios Educación Secundaria, especialidad Matemática - Minedu. https://bit.ly/3rtdqqt

Ministerio de Educación del Perú. (2016). Estrategia nacional de las tecnologías digitales en la educación 2016-2021: de las TIC a la inteligencia digital. https://repositorio.minedu.gob.pe/handle/20.500.12799/5937

Ministerio de Educación del Perú. (2020). Encuesta Nacional Perfil Docente Valorización de la profesión docente Percepción sobre la valoración social de la docencia durante la pandemia (Respuestas múltiples).

Ocańa, Y., Valenzuela, L., y Morillo, J. (2020). La competencia digital en el docente universitario. Propósitos y Representaciones, 8(1), 455. https://doi.org/10.20511/pyr2020.v8n1.455

Perdomo, B., González, O., y Barrutia I. (2020). Competencias digitales en docentes universitarios: una revisión sistemática de la literaturaEDMETIC, 9(2), 92-115. https://doi.org/10.21071/edmetic.v9i2.12796

Rangel, A. (2014). Competencias docentes digitales: propuesta de un perfil. Píxel-Bit, Revista de Medios y Educación, 46, 235-248. https://doi.org/10.12795/pixelbit.2015.i46.15

Sá, M. J., \& Serpa, S. (2020). COVID-19 and the promotion of digital competences in education. COVID-19 y la promoción de las competencias digitales en educación. Universal Journal of Educational Research, 8(10), 45204528. https://doi.org/10.13189/ujer.2020.081020

Trahtemberg, L. (2021). Las redes sociales polarizan y evidencian el fracaso de la educación. https://bit.ly/36P9JSN

Unesco (2020). La educacion en tiempos de pandemia Covid 19. http://www.grade.org.pe/creer/recurso/la-educacionen-tiempos-de-la-pandemia-de-covid-19/

Zárate, A, F., Gurieva, N., \& Arredondo, V. H. J. (2020). The holistic practice of educator digital competencies: Diagnostics and prospective. Pensamiento Educativo, 57(1), 1-16. https://doi.org/10.7764/PEL.57.1.2020.10 